\title{
The importance of continuum eigenstates in electron spectroscopy
}

\author{
D.H. Jakubaßa-Amundsen \\ Physics Section, University of Munich, 85748 Garching, Germany
}

The four characteristic features in the electron spectra from fast ion-atom collisions, the soft electron peak, the cusp, the electron loss peak and the binary encounter peak are strongly influenced by one or both of the atomic (or ionic) potentials acting on the electron, calling for a nonperturbative treatment of the respective final-state interaction. Recent experimental results concerning the peak asymmetry and peak structures are reviewed and interpreted within the available theoretical models.

\section{Introduction}

The spectroscopy of electrons emitted in ion-atom collisions is a sensitive tool to study the electronic structure of the collision partners, and to supply information on the interaction potentials and the collision dynamics [1]. The peak structures in the electron spectra are very sensitive to the underlying theoretical models and hence their detailed comparison with experiment provides a stringent test of theory.

There is a maximum in the doubly differential cross section $\mathrm{d}^{2} \sigma / \mathrm{d} E_{\mathrm{f}} \mathrm{d} \Omega_{\mathrm{f}}$ for electron emission at energies $E_{\mathrm{f}} \rightarrow 0$ (the so-called soft electron (SE) peak). These electrons are caused by direct interaction with the projectile and populate the low continuum states of the target. Conventionally, the first-order Born approximation for direct ionisation [2] has been used for their description, but the new experimental discovery of the peak asymmetry [3] caused by a strong interaction with the projectile field, calls for an improved theoretical model.

Electron spectra recorded at forward emission angles $\left(\vartheta_{\mathrm{f}} \leq 60^{\circ}\right)$ exhibit a second peak near $E_{\mathrm{f}}=$ $2 v^{2} \cos ^{2} \vartheta_{\mathrm{f}}+\epsilon_{\mathrm{i}}^{\mathrm{T}}$ where $v$ is the collision velocity and $\epsilon_{\mathrm{i}}^{\mathrm{T}}$ the initial-state energy of the electron. This is the binary encounter (BE) peak which is basically caused by a binary collision between the projectile nucleus and the active target electron. Since for such fast electrons the final-state interaction with the target field is rather weak, the gross features of the BE peak can be reproduced with the plane wave Bom approximation. However, a series of recent experimental investigations on the precise peak position [4,5] and the discovery of a double-peak structure for very heavy projectiles $[6,7]$ demonstrate that the final-state interaction with the projectile cannot be neglected [8].

When the electrons are recorded in the beam direction $\left(\vartheta_{\mathrm{f}} \approx 0\right)$, a third peak appears at $E_{\mathrm{f}}=v^{2} / 2$, the forward peak or cusp. For bare projectiles, this peak originates from electron capture to the projectile continuum (ECC) and requires the full consideration of the final-state interaction between the electron and the projectile [9]. A proper description of the peak asymmetry is only possible within a higher-order theory [10,11]. For partly stripped fast projectiles another cusp contribution stems from the projectile electrons emitted into low-lying projectile continuum states (ELC). This ELC cusp which is related to the SE peak by a mere frame transformation has a similar asymmetry as the latter. The ELC cusp asymmetry has been confirmed by several experiments [12-14] and can only be explained within a higher-order Born approach for direct ionisation [15].

In electron spectra induced by partly stripped projectiles at larger emission angles $\left(\vartheta_{\mathrm{f}} \geq 10^{\circ}\right)$ the cusp is replaced by the electron loss (EL) peak near $E_{\mathrm{f}}=v^{2} / 2$ $+\epsilon_{\mathrm{i}}^{\mathrm{P}}$ where $\epsilon_{\mathrm{i}}^{\mathrm{P}}$ is the initial energy of the projectile electron. At backward emission angles, this peak is related to the binary encounter peak by a frame transformation [16] and hence shows similar properties as the $B E$ peak when the collision system is reversed. In particular, an anomalous behaviour in the EL peak position and width for very heavy targets has been found [17].

While the mutually related peaks can be treated with the same theoretical models, there is nevertheless a basic difference between the peaks originating from target ionisation and those from electron loss. This difference is due to the fact that the projectiles are usually ionised and hence have at most tightly bound electrons which remain inert during the target ionisation. On the other hand, EL is generally accompanied by a simultaneous ionisation of the loosely bound target valence electrons $[2,18]$. This doubly inelastic process will not explicitly be considered in this work as it does not add essentially new things to the understand- 
ing of the peak properties. The reader is referred to the literature as concerns its calculation within the first-order [2,19] or second-order [20,21] Born approximation.

\section{Theoretical description of the spectral features in comparison with experiment}

The peak structures in the angular differential energy spectra are caused or influenced by the two potentials acting on the electron, namely the parent core field and the field of the perturber atom or ion. Hence, the ejected electron will be in an eigenstate of this two-center potential. While for slow collisions, the molecular orbital theory is well established [22], the use of two-center expansions is rather intricate for high collision velocities, because the strong time-dependence of the potentials requires the consideration of many basis states, including continuum states. Hence, the coupled-channel prescription has up to now basically been used for the calculation of the angular integrated electron spectra [23]. Current theories for electron emission in fast collisions are therefore based on high-energy perturbative approaches which, however, cannot provide a unifying prescription of all the features in the angular differential spectra. We will therefore discuss all spectral features separately within the framework of optimised theories valid in particular regions of the spectra. The interrelation of some of these features allows for a considerable reduction of theoretical efforts required for their interpretation.

\subsection{The forward peak}

The cusp phenomenon which is well established for charged projectiles, is caused by the long-range Coulomb interaction between the ejected electron and the projectile nucleus or ionic core. A correct description must include this interaction nonperturbatively and describe the final electronic state as a projectile continuum eigenstate. In momentum space, this Coulomb wave acquires the following form, when the momentum $\boldsymbol{k}_{\mathrm{f}}$ of the ejected electron approaches the collision velocity $v$ (in atomic units, $\hbar=m=e=1$ )

$$
\begin{aligned}
\varphi_{\boldsymbol{k}_{\mathrm{f}}-\boldsymbol{v}}^{\mathrm{P}}(q) \rightarrow & \frac{Z_{\mathrm{P}}}{\pi^{2} q^{4}} \exp \left(\pi \eta_{\mathrm{f}} / 2\right) \Gamma\left(1+\mathrm{i} \eta_{\mathrm{f}}\right) \\
& \times \exp \left(2 \mathrm{i}\left(Z_{\mathrm{P}} / q\right) \cos \theta_{\boldsymbol{q}, \boldsymbol{k}_{\mathrm{f}}-\boldsymbol{v}}\right), \quad \boldsymbol{k}_{\mathrm{f}} \rightarrow \boldsymbol{v},
\end{aligned}
$$

where $\eta_{\mathrm{f}}=Z_{\mathrm{P}} /\left|\boldsymbol{k}_{\mathrm{f}}-\boldsymbol{v}\right|$ and $Z_{\mathrm{P}}$ is the projectile charge. The prefactor $\exp \left(\pi \eta_{\mathrm{f}} / 2\right) \Gamma\left(1+\mathrm{i} \eta_{\mathrm{f}}\right)$ causes the $\left|\boldsymbol{k}_{\mathrm{f}}-\boldsymbol{v}\right|^{-1}$ divergence of the doubly differential cross section for electron emission. The angular depen- dent phase factor which is discontinuous at $\boldsymbol{k}_{\mathrm{f}}=\boldsymbol{v}$ leads to an asymmetry of the forward peak. The presence of this phase factor makes the theory very sensitive to additional couplings between the electron and the target field, which have to be included within a higher-order approach.

One distinguishes between two classes of theories, one which treats this interaction nonperturbatively in the final state. To this class belongs the continuum distorted wave (CDW) approximation and related approaches which represent the final electronic state by a product of eigenfunctions to projectile and target $[24,25]$. In the CDW approach, the transition amplitude is given by

$a_{\mathrm{fi}}^{\mathrm{CDW}}=-\mathrm{i} \int \mathrm{d} t\left\langle\hat{\psi}_{\boldsymbol{k}_{\mathrm{f}}-\boldsymbol{v}}^{\mathrm{P}} \psi_{\boldsymbol{k}_{\mathrm{f}}}^{\mathrm{T}} \mathrm{e}^{\mathrm{i} \varphi(v)}\left|H-\mathrm{i} \partial_{\mathrm{t}}\right| \chi_{\mathrm{i}}\right\rangle$,

$\psi_{k}(\boldsymbol{\rho}) \equiv \hat{\psi}_{k}(\boldsymbol{\rho}) \exp (\mathrm{i} \boldsymbol{k} \rho), \quad \varphi(\boldsymbol{v})=-\frac{1}{2} \boldsymbol{v r}-\frac{1}{8} v^{2} t$,

where $\psi^{\mathrm{P}}$ and $\psi^{\mathrm{T}}$ denote a projectile and target eigenstate, respectively, $\hat{\psi}_{k}(\boldsymbol{\rho})$ is the distortion part of the continuum wavefunction $\psi_{k}(\rho)$ which multiplies the plane-wave factor $\exp (\mathrm{i} k \rho)$, and $\boldsymbol{r}$ is the electron coordinate with respect to the midpoint between projectile and target. The initial distorted wave $\chi_{\mathrm{i}}$ is also described as a product state of the bound target function (for ECC) or the bound projectile function (for ELC) and a continuum eigenfunction to the other collision partner

$\chi_{\mathrm{i}}= \begin{cases}\psi_{\mathrm{i}}^{\mathrm{P}} \hat{\psi}_{v}^{\mathrm{T}} \exp (\mathrm{i} \varphi(-v)), & \text { for ELC } \\ \psi_{\mathrm{i}}^{\mathrm{T}} \hat{\psi}_{-v}^{\mathrm{P}} \exp (\mathrm{i} \varphi(v)), & \text { for ECC. }\end{cases}$

The transition operator $H-\mathrm{i}_{\mathrm{t}}$ with $H=T+V_{\mathrm{P}}+V_{\mathrm{T}}$ the effective single-particle Hamiltonian describing the electron in the projectile field $\left(V_{\mathrm{P}}\right)$ and the target field $\left(V_{\mathrm{T}}\right)$ induces a coupling through kinetic energy $(T)$ related terms. The full inclusion of the Coulomb fields in initial and final states in this theory may, however, lead to a divergent behaviour of the doubly differential cross section for electron emission [8]. Therefore, one usually makes the eikonal initial state approximation [25] in $\chi_{i}$

$\hat{\psi}_{-v}^{\mathrm{P}}(\boldsymbol{\rho}) \rightarrow \exp \left(-\mathrm{i}\left(Z_{\mathrm{P}} / v\right) \ln (v \rho+\boldsymbol{\nu} \boldsymbol{\rho})\right)$.

This CDW-EIS theory is free of divergencies, while still obeying the correct Coulomb boundary conditions.

The second class of theories disregards the influence of the target field on the final-state electron but includes it - for heavy targets nonperturbatively - in the intermediate electronic states. For electron loss, this is achieved by the strong potential second Born theory $[15,20]$

$a_{\mathrm{fi}}^{\mathrm{SB} 2}=-\mathrm{i} \int \mathrm{d} t\left\langle\psi_{\boldsymbol{k}_{\mathrm{f}}-\nu}^{\mathrm{P}}\left|V_{\mathrm{T}}+V_{\mathrm{T}} G V_{\mathrm{T}}\right| \psi_{\mathrm{i}}^{\mathrm{P}}\right\rangle$ 
In the propagator $G$, the weaker of the two fields $V_{\mathrm{P}}$ and $V_{\mathrm{T}}$ is disregarded. Accordingly, one approximates

$G \rightarrow \begin{cases}G_{\mathrm{P}}, & \text { light target, } \\ G_{\mathrm{T}}, & \text { heavy target, }\end{cases}$

with $G_{\mathrm{P}}=\left(\mathrm{i}_{\mathrm{t}}-T-V_{\mathrm{P}}+\mathrm{i} \epsilon\right)^{-1}$ and $G_{\mathrm{T}}=\left(\mathrm{i} \partial_{\mathrm{t}}-T-\right.$ $\left.V_{\mathrm{T}}+\mathrm{i} \epsilon\right)^{-1}$.

For electron capture to continuum, $\psi_{\mathrm{i}}^{\mathrm{P}}$ is replaced by a target state $\psi_{\mathrm{i}}^{\mathrm{T}}$ and correspondingly the adjacent $V_{\mathrm{T}}$ by $V_{\mathrm{p}}$. This leads to the impulse approximation $[11,26]$, which first had been developed for bound-state capture [27]

$a_{\mathrm{fi}}^{\mathrm{IA}}=-\mathrm{i} \int \mathrm{d} t\left\langle\psi_{\boldsymbol{k}_{\mathrm{f}}-\boldsymbol{v}}^{\mathrm{P}}\left|V_{\mathrm{T}}+V_{\mathrm{T}} G V_{\mathrm{P}}\right| \psi_{\mathrm{i}}^{\mathrm{T}}\right\rangle$,

with $G$ from Eq. (6). The substitution $G \rightarrow G_{\mathrm{P}}$ is termed "post IA" while $G \rightarrow G_{\mathrm{T}}$ leads to the "prior IA". In the two theories for ELC and ECC, Eqs. (5) and (7), an on-shell approximation is made in the intermediate states.

The second class of cusp theories which gives a better account of the interaction zone at the expense of violating the Coulomb boundary conditions is very satisfactory in describing details of the cusp shape $[11,15]$. As an example, Fig. 1 shows the ELC cusp from $1.5 \mathrm{MeV} \mathrm{He}^{+}$on $\mathrm{Xe}$. The calculation is performed with the SB2 $\left(G \rightarrow G_{\mathrm{T}}\right)$ which has been averaged over the acceptance angle of the detector $\left(\theta_{0}=\right.$ $1.28^{\circ}$ ). The data [28] have not been measured on an absolute scale, and hence are normalised to theory.

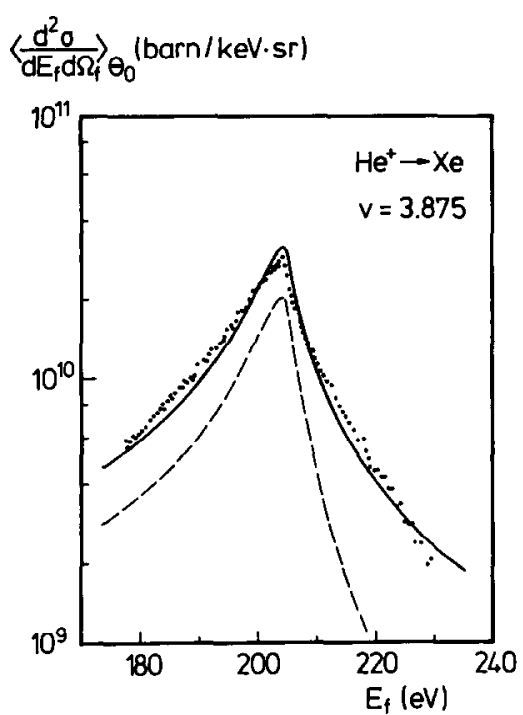

Fig. 1. Doubly differential ELC cusp cross section for 1.5 $\mathrm{MeV} \mathrm{He}^{+}+\mathrm{Xe}$ as a function of electron energy: $\bullet$, experiment $[14,28]$ normalised to theory; -, SB2 theory [15], including the doubly inelastic contribution via first Born; ----, singly inelastic contribution.

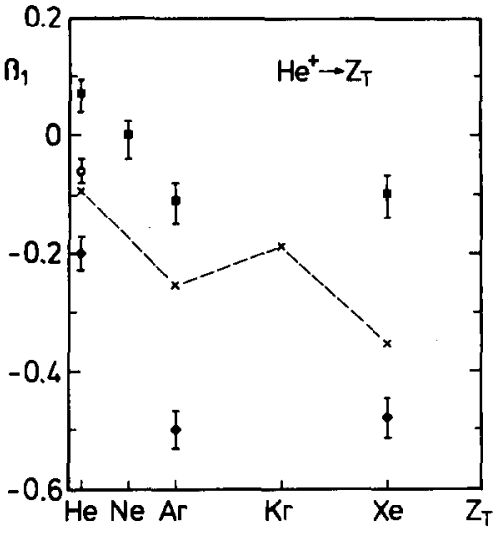

Fig. 2. Cusp shape parameter $\beta_{1}$ for ELC from $0.4 \mathrm{MeV} / \mathrm{amu}$ $\mathrm{He}^{+}$as a function of target chargc. Experiment: •, Jensen [12]; ஐ, Atan et al. [14] and Lucas [28]; O, Gulyás et al. [29]. Theory: $\times$, SB2, including the doubly inelastic contribution to second order (for $\mathrm{He}$, ref. [20]) and to first order (for the heavier targets, ref. [15]). The lines are an eye-guide.

Comparison of the cusp shape parameter $B_{00}$ for Xe and $\mathrm{Ar}$ with absolute data $[12,14,15]$ indicates that the SB2 theory slightly overestimates experiment (within a factor of 2). The cusp shape parameters are defined through the expansion of the doubly differential cross section in terms of electron momentum $\kappa_{\mathrm{f}}=\boldsymbol{k}_{\mathrm{f}}-\boldsymbol{v}$ and ejection angle $\theta_{\mathrm{f}}^{\prime}$ in the projectile reference frame

$\frac{\mathrm{d}^{2} \sigma}{\mathrm{d} E_{\mathrm{f}} \mathrm{d} \Omega_{\mathrm{f}}}=\frac{k_{\mathrm{f}}}{\kappa_{\mathrm{f}}} \sum_{n, l=0}^{\infty} B_{n l} \kappa_{\mathrm{f}}^{n} P_{l}\left(\cos \theta_{\mathrm{f}}^{\prime}\right)$,

with $E_{\mathrm{f}}=k_{\mathrm{f}}^{2} / 2$ and $P_{l}$ a Legendre polynomial. Fig. 2 depicts the target dependence of the shape parameter $\beta_{1}=B_{01} / B_{00}$ (which is a measure for the cusp asymmetry) in the case of $0.4 \mathrm{MeV} / \mathrm{amu} \mathrm{He}{ }^{+}$impact. Unfortunately, the $\beta_{1}$ data extracted at different laboratories $[12,14,28,29]$ for a given target differ strongly from each other. This may be related to the sensitivity of $\beta_{1}$ to small features in the spectra close to the cusp maximum which are strongly influenced by the detector transmission function, but may have only a minor physical meaning. Although this large spread of the $\beta_{1}$ data casts some doubt on the significance of a $B_{n l}$ expansion, the data (from a given laboratory) as well as theory $[15,20]$ clearly exhibit the tendency of an increased asymmetry when passing from light to heavy targets.

\subsection{The soft electron peak}

Electrons of very low energy are strongly influenced by the target field. Their final state has therefore to be described by a target continuum eigenstate. In order to show that the soft electron peak is closely related to the electron loss cusp, the doubly differential ELC 
cross section is transformed into the projectile frame of reference (denoted by primed quantities)

$$
\left(\frac{\mathrm{d}^{2} \sigma^{E L C}}{\mathrm{~d} E_{\mathrm{f}}^{\prime} \mathrm{d} \Omega_{\mathrm{f}}^{\prime}}\right)_{\text {p.f. }}=\frac{\left|\boldsymbol{k}_{\mathrm{f}}-\boldsymbol{v}\right|}{k_{\mathrm{f}}} \frac{\mathrm{d}^{2} \sigma^{\text {ELC }}}{\mathrm{d} E_{\mathrm{f}} \mathrm{d} \Omega_{\mathrm{f}}} .
$$

From this equation it is immediately seen that the prefactor $\left|\boldsymbol{k}_{\mathrm{f}}-\boldsymbol{v}\right|$ cancels the divergence in the Coulomb wave (Eq. (1)) such that the projectile frame cross section is finite. The $\mathrm{SE}$ cross section is then simply obtained by interchanging projectile and target, and by replacing $\boldsymbol{v}$ by

$$
\frac{\mathrm{d}^{2} \sigma^{\mathrm{SE}}}{\mathrm{d} E_{\mathrm{f}} \mathrm{d} \Omega_{\mathrm{f}}}=\left.\left(\frac{\mathrm{d}^{2} \sigma^{\mathrm{ELC}}}{\mathrm{d} E_{\mathrm{f}}^{\prime} \mathrm{d} \Omega_{\mathrm{f}}^{\prime}}\right)_{\text {p.f. }}\right|_{\substack{\text { projectile target } \\ \text { prot. }}}
$$

In a higher-order approach, also the role of projectile and target has to be interchanged, i.e. the ELC theory for a light projectile and a heavy target corresponds to the SE theory for a heavy projectile and a light target. Although Eq. (10) with Eq. (9) implies that the SE cross section is finite at $E_{\mathrm{f}}=0$, the asymmetry from the phase in Eq. (1) persists. Measurements of the SE peak shape are much more difficult than those of the ELC cusp shape because the energy of the electrons is in the $\mathrm{eV}$ region only (as compared to hundreds of $\mathrm{eV}$ for the ELC cusp), which requires extremely thin targets [3,30]. Fig. 3 shows the first measurement of the SE cusp asymmetry for $106 \mathrm{keV} / \mathrm{amu} \mathrm{H}^{+}$and ${ }^{3} \mathrm{He}^{2+}$ colliding with $\mathrm{Ne}$ [30]. The influence of the projectile field skews the SE peak to the high-energy side (i.e. emission angle $\vartheta_{f}=0$ ), whereas the ECC cusp is skewed to the low-energy side. So far, a qualitative theoretical interpretation has only been given within the CDW-EIS approach $[25,30]$.

\subsection{The binary encounter peak}

The binary encounter peak originates from target electrons undergoing a quasielastic collision with the

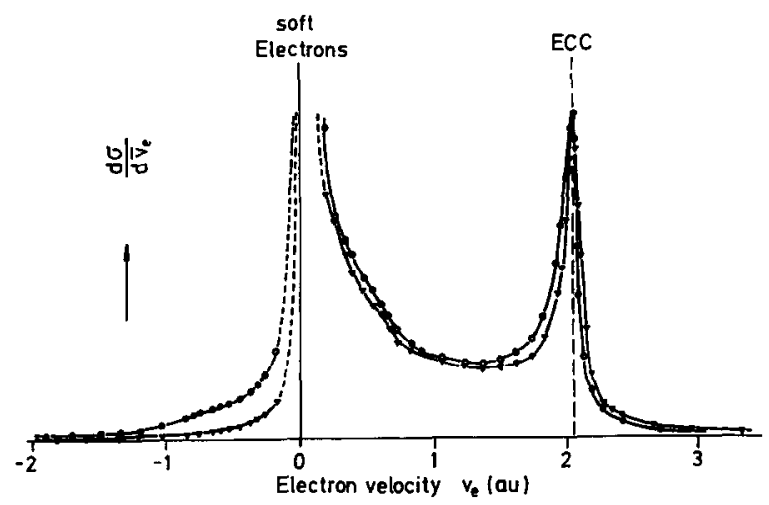

Fig. 3. Differential cross section $d \sigma / d k_{\mathrm{f}}$ for soft electron emission from $\mathrm{Ne}$ by $106 \mathrm{keV} / \mathrm{amu} \mathrm{H}^{+}$impact $(O)$ and

${ }^{3} \mathrm{He}^{2+}$ impact $(\nabla)$ at $\vartheta_{\mathrm{f}}=0$ (corresponding to $k_{\mathrm{f}}=v_{\mathrm{e}}>0$ ) and $\vartheta_{\mathrm{f}}=180^{\circ}\left(k_{\mathrm{f}}<0\right)$. Taken from Suárez et al. [30]

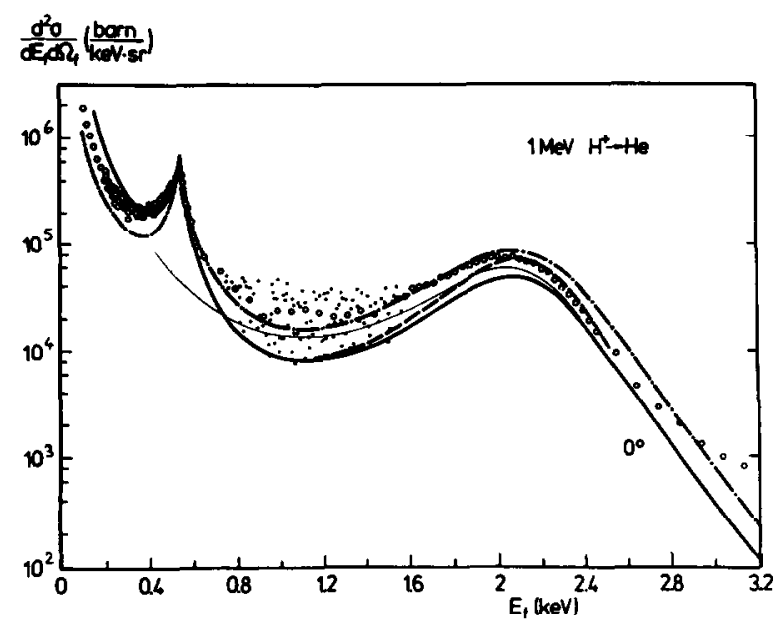

Fig. 4. Doubly differential cross section for electron emission in $1 \mathrm{MeV} \mathrm{H}^{+}+\mathrm{He}$ collisions at $\vartheta_{\mathrm{f}}=\mathbf{0}$ as a function of electron energy $E_{\mathrm{f}}$. Experiment (acceptance angle $\theta_{0}=1.67^{\circ}$ ): $O$, data averaged over a certain energy interval; $\bullet$, selection of not averaged data points from Fiedler et al. [32]. Theory (averaged over $\theta_{0}$ in the cusp region): thick solid line, transverse peaked post IA [11]; -----, fully peaked prior IA [26]; --- , CDW-EIS [31]; thin solid line, PWBA with a Roothaan-Hartree-Fock target function.

projectile. The final-state influence of the target field is of minor importance, and hence the simplest prescription is the plane wave Born approximation where the outgoing electron is described by a plane wave:

$$
\begin{aligned}
& \frac{\mathrm{d}^{2} \sigma^{\text {PWBA }}}{\mathrm{d} E_{\mathrm{f}} \mathrm{d} \Omega_{\mathrm{f}}}=\frac{2 \pi k_{\mathrm{f}}}{v} N_{i} \int \mathrm{d} \boldsymbol{q} \\
& \left|\tilde{V}_{\mathrm{P}}(q)\right|^{2}\left|\varphi_{\mathrm{i}}^{\mathrm{T}}\left(k_{\mathrm{f}}-q\right)\right|^{2} \delta\left(E_{\mathrm{f}}-\epsilon_{\mathrm{i}}^{\mathrm{T}}-\boldsymbol{q} v\right) .
\end{aligned}
$$

$\bar{V}_{\mathrm{P}}$ and $\varphi_{\mathrm{i}}^{\mathrm{T}}$ are the Fourier transforms of the projectile field and the target bound state, respectively, and $N_{i}$ is the number of target electrons in the state $i$. The gross features of the $\mathrm{BE}$ peak (except for heavy structured projectiles) are well described by the PWBA: The peak position near $E_{\mathrm{f}}=2 v^{2} \cos ^{2} \vartheta_{\mathrm{f}}+\epsilon_{\mathrm{i}}^{\mathrm{T}}$ is determined by the $\delta$-function and the peak shape is related to the boundstate momentum distribution (with maximum at $\boldsymbol{q}=\boldsymbol{k}_{\mathrm{f}}$ ). However, a detailed investigation of the BE peak shows the influence of the projectile field (and to a lesser extent also of the target field) on the ejected electron. The fields cause a change in intensity and a shift of the peak maximum to lower energies [4,5,8,31]. Fig. 4 gives a comparison between experiment [32] and various theoretical approaches for the simplest collision system investigated, $1 \mathrm{MeV} \mathrm{H}^{+}+\mathrm{He}$. The best description of the data over a wide energy regime is provided by the prior impulse approximation, whereas the PWBA and the post IA give too low intensities in the binary encounter region. The CDW-EIS theory gives the correct peak intensity but a too high peak position. 
For heavy projectiles, the first Born treatment of the projectile interaction in Eq. (11) becomes inadequate. Theory can readily be improved by noting that the projectile field in momentum space is related to the first Born approximation $f^{\mathrm{B} 1}(q)$ of the elastic scattering amplitude for an electron from the projectilc

$$
\tilde{V}_{\mathrm{P}}(q)=-(2 \pi)^{-1 / 2} f^{\mathrm{B} 1}(q)
$$

where $q$ is the momentum transfer. The full electronprojectile coupling can readily be accounted for by replacing $f^{\mathbf{B} 1}(\boldsymbol{q})$ with the exact scattering amplitude $f\left(\boldsymbol{k}_{\mathrm{f}}-\boldsymbol{v}-\boldsymbol{q}, \boldsymbol{k}_{\mathrm{f}}-\boldsymbol{v}\right)$ which not only depends on $\boldsymbol{q}$, but also on the momentum of the scattering electron in the projectile frame, $\boldsymbol{k}_{\mathrm{f}}-\boldsymbol{v}$. Then one arrives at the quantum mechanical version of the elastic scattering model [33], the so-called electron impact approximation (EIA) [34]

$$
\begin{aligned}
\frac{\mathrm{d}^{2} \sigma^{\text {EIA }}}{\mathrm{d} E_{\mathrm{f}} \mathrm{d} \Omega_{\mathrm{f}}}= & \frac{k_{\mathrm{f}}}{v} N_{i} \int \mathrm{d} \boldsymbol{q}\left|\int\left(\boldsymbol{k}_{\mathrm{f}}-v-\boldsymbol{q}, \boldsymbol{k}_{\mathrm{f}}-v\right)\right|^{2} \\
& \times\left|\varphi_{\mathrm{i}}^{\mathrm{T}}\left(\boldsymbol{k}_{\mathrm{f}}-q\right)\right|^{2} \delta\left(E_{\mathrm{f}}-\epsilon_{\mathrm{i}}^{\mathrm{T}}-q v\right) .
\end{aligned}
$$

This theory is often termed impulse approximation $[4,35]$, which is misleading because it includes only the first-order term in the IA, Eq. (17), and not the second-order term. In the case of bare projectiles, Eq. (13) is equal to the Born approximation (11) because

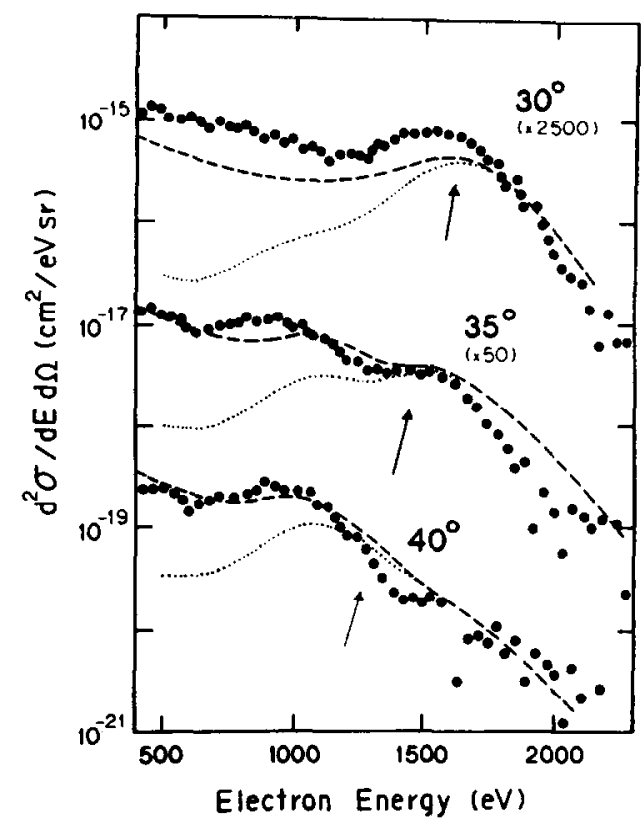

Fig. 5. Doubly differential cross section for electron ejection from $1 \mathrm{MeV} / \mathrm{amu} \mathrm{U}^{2 \mathrm{l}}$ on $\mathrm{He}$ as a function of electron energy at the angles $\vartheta_{\mathrm{f}}=30^{\circ}, 35^{\circ}$ and $40^{\circ}$. $\bullet$, experiment, normalised to theory; $\cdots \cdots$. EIA theory; ---- EIA theory plus background from distant collisions, calculated with the CTMC method. The arrows indicate the energy $E_{\mathrm{f}}=$ $2 v^{2} \cos ^{2} \vartheta_{\mathrm{f}}+\epsilon_{\mathrm{i}}^{\mathrm{T}}$. Taken from Reinhold et al. [37].

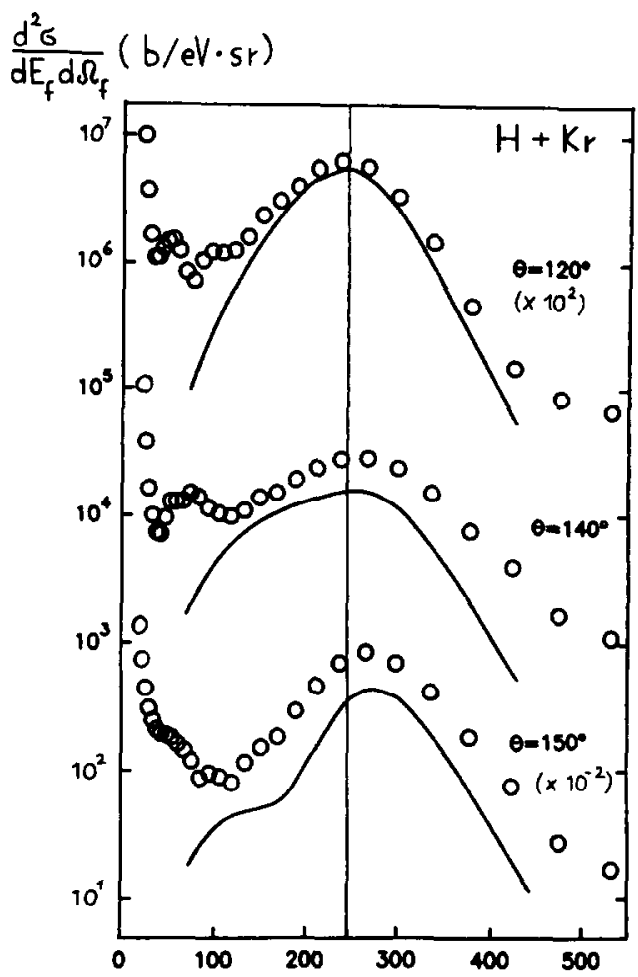

Fig. 6. Doubly differential cross section for electron emission in $0.5 \mathrm{MeV} \mathrm{H}+\mathrm{Kr}$ collisions at $\vartheta_{\mathrm{f}}=120^{\circ}, 140^{\circ}$ and $150^{\circ}$. $\circ$, Kuzel et al. [17], singles data; - , EIA for electron loss, singly inelastic contribution. The vertical line denotes $E_{\mathrm{f}}=v^{2} / 2+$ $\epsilon_{\mathrm{i}}^{\mathrm{P}}$.

for a Coulomb field, the first Born approximation to $f$ gives the correct modulus of the exact scattering amplitude. This explains the success of the Born theory even for heavy projectiles. For partly stripped projectiles, on the other hand, $f$ can be very different from $f^{\mathrm{B} 1}$, particularly for heavy projectiles with many electrons. Like in the case of elastic electron scattering on heavy neutral atoms [36], the angular dependent differential electron-ion scattering cross section shows sharp Ramsauer minima at certain scattering angles which vary with impact energy. These structures in $f$ strongly influence the shape of the binary encounter peak. Fig. 5 shows the change of the BE peak shape with angle for $1 \mathrm{MeV} / \mathrm{amu} \mathrm{U} \mathrm{U}^{21+}$ on He between $30-40^{\circ}$. Whereas the first Born theory (Eq. (11)) predicts a continuous shift of the peak maximum with angle, the Ramsauer effect leads to a discontinuous peak shift accompanied by a double-peak structure around $35^{\circ}$. This structure is clearly seen in the experimental spectra and can qualitatively be described by the above theory [37].

\subsection{The electron loss peak}

The electron loss peak for large emission angles is closely related to the binary encounter peak for the 
reversed collision system. In complete correspondence to Eq. (9), the two phenomena are related by a simple transformation from the projectile frame to the target frame of reference

$$
\frac{\mathrm{d}^{2} \sigma^{\mathrm{EL}}}{\mathrm{d} E_{\mathrm{f}} \mathrm{d} \Omega_{\mathrm{f}}}=\left.\frac{k_{\mathrm{f}}}{\left|\boldsymbol{k}_{\mathrm{f}}-v\right|}\left(\frac{\mathrm{d}^{2} \sigma^{\mathrm{BE}}}{\mathrm{d} E_{\mathrm{f}}^{\prime} \mathrm{d} \Omega_{\mathrm{f}}^{\prime}}\right)_{\mathrm{p} . \mathrm{f} .}\right|_{\substack{p \rightarrow-v \\ \text { projectile } \rightleftarrows \text { target }}},
$$

if the collision system is reversed (i.e. the role of projectile and target interchanged) and the velocity $v$ replaced by $-v$. Extracting the properties of the BE electrons from Eq. (11), one easily finds the position of the EL peak,

$E_{\mathrm{f}}=\epsilon_{\mathrm{i}}^{\mathrm{P}}+v^{2} / 2$,

as well as the connection between the emission angles for the binary encounter electrons $\left(\vartheta_{\mathrm{f}}^{\mathrm{BE}}\right)$ and for the EL electrons $\left(\vartheta_{\mathrm{f}}^{\mathrm{EL}}\right)$, both defined with respect to $v$ (i.e. in the target frame)

$\vartheta_{\mathrm{f}}^{\mathrm{EL}}=\pi-2 \vartheta_{\mathrm{f}}^{\mathrm{BE}}$.

In Eq. (16), the initial-state energy $\epsilon_{i}^{\mathrm{P}}$ has been neglected. From the interrelation (14) it follows that for heavy target atoms, the Ramsauer structures will also influence the electron loss peak. In Fig. 6 electron loss spectra from $0.5 \mathrm{MeV} \mathrm{H}$ on krypton at backward angles around $140^{\circ}$ are shown. For this system, the

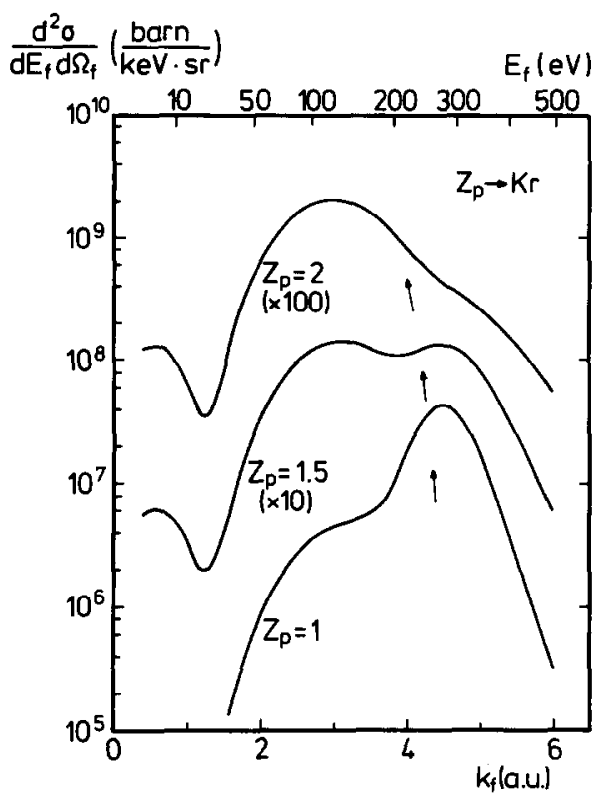

Fig. 7. Doubly differential cross section for electron emission in $0.5 \mathrm{MeV} / \mathrm{amu} Z_{\mathrm{P}}+\mathrm{Kr}$ at $\vartheta_{\mathrm{f}}=150^{\circ}$ (one-electron projectiles with nuclear charge $1,1.5$ and 2). Calculated with the EIA, singly inelastic contribution. The arrows mark $E_{\mathrm{f}}=$ $k_{\mathrm{f}}^{2} / 2=v^{2} / 2+\epsilon_{\mathrm{i}}^{\mathrm{P}}$.

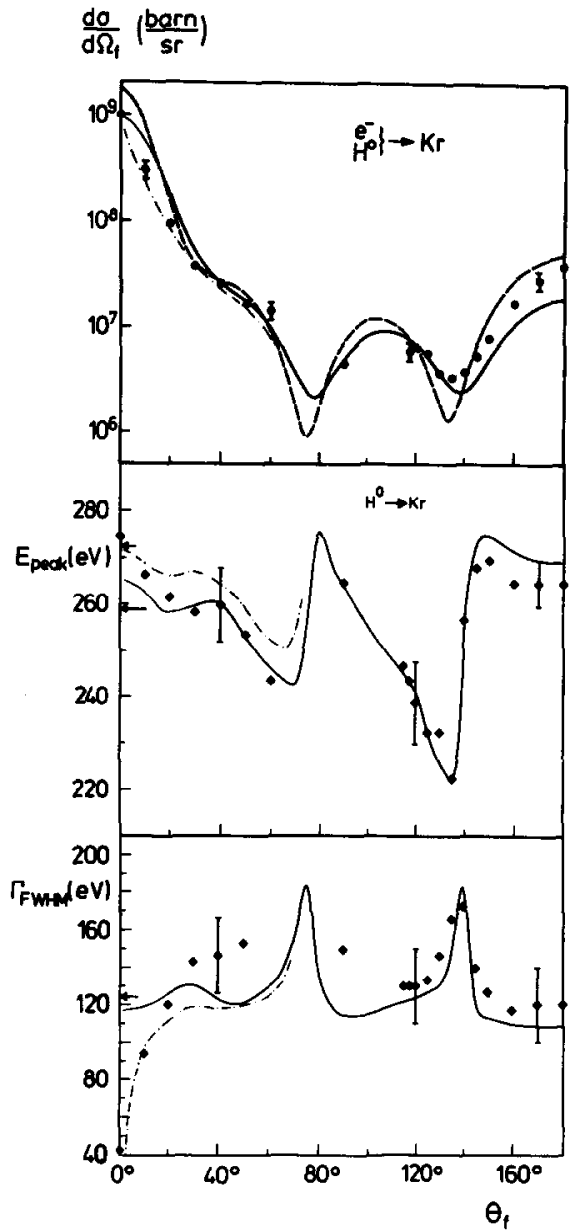

Fig. 8. (Top): Differential cross section for elastic electron scattering on $\mathrm{Kr}$ at energy $v^{2} / 2(---) ; \mathrm{d}^{2} \sigma / \mathrm{d} E_{\mathrm{f}} \mathrm{d} \Omega_{\mathrm{f}}$ for electron loss from $0.5 \mathrm{MeV} \mathrm{H}+\mathrm{Kr}$, integrated over the energy interval (0.15-0.4) keV as function of emission angle $\vartheta_{\mathrm{f}}(-$, -.--); Experiment (•): Singles data from Kuzel et al. [17], normalised to theory. For absolute cross sections, all data points should be multiplied by 1.27. (Middle): position of the EL peak maximum. The arrows denote $E_{\text {peak }}=v^{2} / 2(\leftarrow)$ and $v^{2} / 2+\epsilon_{\mathrm{i}}^{\mathrm{P}}(\longleftarrow)$. (Bottom): full width at half maximum of the EL peak. The arrow denotes the width corresponding to the Compton profile. Experiment $(\bullet)$ : singles data for 0.5 $\mathrm{MeV} \mathrm{H}+\mathrm{Kr}$ from Kuzel et al. [17]. Theory: -, EIA for electron loss; --.--, peaked strong potential second Born theory for electron loss [15], singly inelastic contribution.

effects from the Ramsauer minimum at $135^{\circ}$ for the $\mathbf{K r}$ target [36] are hidden, but they become prominent if the projectile charge is artificially increased to $Z_{\mathrm{P}}=1.5$ (Fig. 7), causing a double-peak structure as for the binary encounter peak in reversed collision systems. This double-peak structure is a type of resonance effect which can be observed upon varying the projectile velocity, the projectile charge or the emission angle 
such that the width of the structures in the scattering amplitude $f$ entering into the loss cross section

$$
\begin{aligned}
\left(\frac{\mathrm{d}^{2} \sigma^{\mathrm{EIA}}}{\mathrm{d} E_{\mathrm{f}} \mathrm{d} \Omega_{\mathrm{f}}}\right)^{\mathrm{EL}}= & \frac{k_{\mathrm{f}}}{v} N_{i} \int \mathrm{d} \boldsymbol{q}\left|f\left(q+v, k_{\mathrm{f}}\right)\right|^{2} \\
& \times\left|\varphi_{\mathrm{i}}^{\mathrm{P}}(q)\right|^{2} \delta\left(E_{\mathrm{f}}-\epsilon_{\mathrm{i}}^{\mathrm{P}}-v^{2} / 2-q v\right),
\end{aligned}
$$

matches the width of the bound electron's momentum distribution $\varphi_{i}^{\mathrm{P}}$ [38]. However, although in the $\mathrm{H}+\mathrm{Kr}$ system the Ramsauer structures are not clearly visible in the spectra, they cause large variations in the position and width of the electron loss peak near those angles which are related to minima in the differential cross section for elastic electron scattering on $\mathrm{Kr}$ (Fig. 8). The EIA, Eq. (17), used for their theoretical interpretation is only valid for emission angles above $\sim 20^{\circ}$ where the influence of the target field on the ejected electron dominates. For smaller emission angles the strong potential second Born theory (Eq. (5)) has to be used. A peaking version of the SB2, as first introduced by Hartley and Walters [39], is well able to describe the increase in peak energy and the decrease in width for $\vartheta_{\mathrm{f}} \rightarrow 0$ which is seen in experiment [17].

\section{Conclusion}

A series of examples has been given which demonstrate the importance of a proper description of the electronic states for the interpretation of angular differential electron spectra. In particular, for certain collision systems (e.g. ionisation of a light atom or ion by a heavy collision partner) or regions of the spectra (e.g. the cusp region) the ionisation process cannot be treated by a perturbative description of the interaction potentials, even at high collision velocities. The gross structures in the spectra are reproduced upon selecting the final electronic continuum state as eigenstate to the stronger of the two interaction potentials in that particular region of the spectrum. However, details of the electron distribution like precise peak position and shape can often only be properly described by a higher-order theory which allows the electron to propagate in intermediate eigenstates of the heavier collision partner, which for fast collisions are lying in the continuum. If the interaction potential is not purely Coulombic but modified by core electrons, the electronic states have to be chosen as exact scattering eigenstates, rather than hydrogenlike approximations. This is essential for the ECC cusp in case of partly stripped projectiles, as well as for the EL peak at backward angles and the $\mathrm{BE}$ peak in the case of structured heavy collision partners. Unfortunately, no unifying theory is existing at presence which can describe all details in the angular differential energy spectra to high accuracy.

\section{Acknowledgements}

This work includes results from the FrankfurtMunich-Brighton collaboration and I should particularly like to thank K.O. Groeneveld, M. Kuzel and M.W. Lucas for many fruitful discussions and for a close look into the experiments. Support from the GSI Darmstadt is gratefully acknowledged.

\section{References}

[1] M.E. Rudd and J.H. Macek, Case Stud. At. Phys. 3, eds. E.W. McDaniel and M.R.C. McDowell (North-Holland, Amsterdam, 1972) p. 47.

[2] D.R. Bates and G. Griffing, Proc. Phys. Soc. London A 67 (1954) 663.

[3] S. Suárez, C. Garibotti, W. Meckbach and G. Bernardi, Phys. Rev. Lett. 70 (1993) 418.

[4] D.H. Lee, P. Richard, T.J.M. Zouros, J.M. Sanders, J.L. Shinpaugh and H. Hidmi, Phys. Rev. A 41 (1990) 4816.

[5] A.D. Gonzales, P. Dahl, P. Hvelplund and P.D. Fainstein, J. Phys. B 26 (1993) L135.

[6] C. Kelbch, R.E. Olson, S. Schmidt, H. Schmidt-Böcking and S. Hagmann, Phys. Lett. A 139 (1989) 304.

[7] W. Wolff; J.L. Shinpaugh, H.E. Wolf, R.E. Olson, J. Wang, S. Lencinas, D. Piscevic, R. Herrmann and $H$. Schmidt-Böcking, J. Phys. B 25 (1992) 3683.

[8] M. Brauner and J.H. Macek, Phys. Rev. A 46 (1992) 2519.

[9] J. Macek, Phys. Rev. A 1 (1970) 235.

[10] K. Dettmann, K.G. Harrison and M.W. Lucas, J. Phys. B 7 (1974) 269.

[11] D.H. Jakubaßa-Amundsen, J. Phys. B 16 (1983) 1767.

[12] K.E. Jensen, Thesis, University of Aarhus, Denmark, (1986).

[13] Á. Kövér, L. Sarkadi, J. Pálinkás, D. Berényi, Gy. Szabú, T. Vajnai, O. Heil, K.O. Groeneveld, J. Gibbons and I.A. Sellin, J. Phys. B 22 (1989) 1595.

[14] H. Atan, W. Steckelmacher and M.W. Lucas, J. Phys. B 23 (1990) 2579.

[15] D.H. Jakubaßa-Amundsen, J. Phys. B 26 (1993) 2853.

[16] F. Drepper and J.S. Briggs, J. Phys. B 9 (1976) 2063.

[17] M. Kuzel, R. Maier, O. Heil, D.H. Jakubaßa-Amundsen, M.W. Lucas and K.O. Groeneveld, Phys. Rev. Lett. 71 (1993) 2879.

[18] E.C. Montenegro, W.S. Melo, W.E. Meyerhof and A.G. de Pinho, Phys. Rev. Lett. 69 (1992) 3033.

[19] M.H. Day, J. Phys. B 14 (1981) 231.

[20] D.H. Jakubaßa-Amundsen, J. Phys. B 23 (1990) 3335.

[21] J. Wang, C.O. Reinhold and J. Burgdörfer, Phys. Rev. A 45 (1992) 4507.

[22] U. Wille and R. Hippler, Phys. Rep. 132 (1986) 129. 
[23] N. Toshima, J. Phys. B 25 (1992) L635.

[24] I.M. Cheshire, Proc. Phys. Soc., London 84 (1964) 89.

[25] P.D. Fainstein, V.H. Ponce and R.D. Rivarola, J. Phys. B 24 (1991) 3091.

[26] D.H. Jakubaßa-Amundsen, Phys. Rev. A 38 (1988) 70.

[27] J.S. Briggs, J. Phys. B 10 (1977) 3075.

[28] M.W. Lucas, private communication, (1992).

[29] L. Gulyás, Gy. Szabó, D. Berényi, Á. Kövér, K.O. Groeneveld, D. Hofmann and M. Burkhard, Phys. Rev. A 34 (1986) 2751.

[30] S. Suárez, C. Garibotti, G. Bernardi, P. Focke and W. Meckbach, Phys. Rev. A 48 (1993) 4339.

[31] P.D. Fainstein, V.H. Ponce and R.D. Rivarola, Phys. Rev. A 45 (1992) 6417.
[32] C. Fiedler, M. Kuzel and K.O. Groencveld, private communication, (1993).

[33] D. Burch, H. Wieman and W.B. Ingalls, Phys. Rev. Lett. 30 (1973) 823.

[34] D.H. Jakubaßa, J. Phys. B 13 (1980) 2099.

[35] D.R. Schultz and R.E. Olson, J. Phys. B 24 (1991) 3409.

[36] I.E. McCarthy, C.J. Noble, B.A. Phillips and A.D. Turnbull, Phys. Rev. A 15 (1977) 2173.

[37] C.O. Reinhold, D.R. Schultz, R.E. Olson, C. Kelbch, R. Koch and H. Schmidt-Böcking, Phys. Rev. Lett. 66 (1991) 1842.

[38] D.H. Jakubaßa-Amundsen, J. Phys. B 26 (1993) L227.

[39] H.M. Hartley and H.R.J. Walters, J. Phys. B 20 (1987) 3811 . 